\title{
La Pedagogía Hospitalaria y los Derechos del Niño: \\ un camino recorrido y por recorrer
}

\author{
Asensio-Ramón, Patricia \\ Universitat de València, Valencia, España \\ patricia.asensio@uv.es
}

\section{Resumen}

En las últimas décadas y, debido al proceso de humanización que se ha estado llevando a cabo dentro del ámbito de la medicina en los últimos tiempos, se ha modificado el modelo de atención del niño enfermo y hospitalizado permitiendo que el ámbito educativo forme parte de su atención integral, del niño enfermo y hospitalizado, mejorando así su calidad de vida.

La Pedagogía Hospitalaria es la disciplina que da respuesta a las necesidades educativas, afectivas y sociales de los menores enfermos. Es una de las ramas más desconocida de la pedagogía por haber estado hasta ahora muy tecnificado el ámbito sanitario y, carecer de una presencia consolidada en los estudios de Grado en la Universidad. Actualmente, su actividad está yendo más allá de la planta de pediatría aunque, sí que es cierto que sus funciones se han llevado a cabo hasta ahora principalmente en las aulas hospitalarias.

Gracias a esta disciplina y a sus unidades escolares se han podido hacer realidad derechos establecidos y reflejados en Declaraciones Internacionales, así como en documentos oficiales relacionados con el sector educativo-sanitario.

Nuestro planteamiento se ha fundamentado en conocer la Pedagogía Hospitalaria, sus Aulas Hospitalarias, su historia, sus objetivos, pero, fundamentalmente, en conocer aquella normativa que la regula estableciendo una serie de derechos que tiene todo niño enfermo y, que tienen que reflejarse tanto en las normas a nivel de documento como en las acciones llevadas a cabo en la realidad.

A través de esta comunicación se va a realizar un análisis cualitativo de esas declaraciones que destacan uno de los derechos más importantes como es el Derecho a la Educación, así como una serie de documentos oficiales que han regulado la Pedagogía Hospitalaria.

\section{Abstract}

In recent decades and due to the humanization process that has been taking place within the field of medicine in recent times, the model of care for the sick and hospitalized child has been modified, allowing the education field to be part of its comprehensive care, of the sick and hospitalized child thus improving their quality of life.

Hospital Pedagogy is the discipline that responds to the educational, affective and social needs of sick children. It is one of the most unknown branches of pedagogy because it has been highly technified until now in the health field, and lacks a consolidated presence in undergraduate studies at the University.Currently, its activity is going beyond the pediatric facility although, yes, it is true that its functions have been carried out so far mainly in the hospitaller classrooms.

Thanks to this discipline and its school units, rights established and reflected in International Declarations have been made possible, as well as in official documents related to the education-health sector.

Our approach has been based on knowing the Hospital Pedagogy, its hospitaller classrooms, its history, its objectives, but, fundamentally, to know that regulation that regulates it establishing a series of rights that every sick child has, and that have to be reflected so much in the norms at the document level as in the actions carried out in reality.

Through this communication, a qualitative analysis of these Declarations will be made, highlighting one of the most important rights such as the Right to Education, as well as a series of official documents that have regulated Hospital Pedagogy.

Palabras clave: Pedagogía Hospitalaria, Derechos del Niño.

Keywords: Hospital Pedagogy, Child Rights. 


\section{INTRODUCCIÓN}

La Pedagogía Hospitalaria es una disciplina que acoge las unidades escolares ubicadas en las instituciones hospitalarias denominadas, aulas hospitalarias. Según Molina (2017), la UNESCO dispone de muchos informes que la avalan, mediante ese derecho de todos a la educación mostrando la oportunidad de ser educados. Es la disciplina que previene repercusiones negativas respecto a la enfermedad y mejora la calidad de vida de las personas enfermas o ingresadas en el hospital.

Los niños enfermos son niños, es decir, quieren jugar, reír llorar, soñar, etcétera. Por este motivo, en todos los hospitales españoles se está llevando a cabo un Programa de Humanización para los cuidados del niño hospitalizado y así hacer más llevadero su ingreso. Por otra parte, no debemos olvidar que tienen que ser escuchados y necesitan sentirse importantes y felices siendo el humor, la clave en el proceso de recuperación y en su educación (Calvo, 2017).

\section{Objetivos}

Conocer la disciplina Pedagogía Hospitalaria, sus aulas hospitalarias, pero sobre todo conocer los documentos y Declaraciones Internacionales que la regulan estableciendo una serie de derechos que tiene todo escolar enfermo, entre ellos, el Derecho a la Educación.

\section{Metodología}

Con esta comunicación se ha realizado un análisis cualitativo de declaraciones y documentos que destacan derechos tan importantes del niño enfermo u hospitalizado entre ellos, el Derecho a la Educación. Se han resaltado a su vez documentos oficiales que han regulado la Pedagogía Hospitalaria.

\section{MARCO TEÓRICO}

Todo niño hospitalizado es un sujeto vulnerable, frágil, que requiere cuidados y atención de los familiares y agentes sociales que le rodean. Hemos de paliar sus necesidades, sus sufrimientos, así como descubrir sus posibilidades existenciales dentro de la personalidad que puede desarrollar. Es evidente, que en el contexto hospitalario siempre se deben asumir las necesidades del otro y el desarrollo máximo de sus posibilidades (Torralba, 2002).

Profundizando en dicha disciplina mostramos una de las definiciones conceptuales de Lizasoáin (2000), enfocándola como una «Rama diferencial de la pedagogía que se encarga de la educación del niño enfermo y hospitalizado, de manera que no se retrase en su desarrollo personal ni en sus aprendizajes, a la vez que procura atender a las necesidades psicológicas y sociales generadas como consecuencia de la hospitalización y de la concreta enfermedad que padece» (p.110). Según Molina (2017), la UNESCO dispone de muchos informes que la avalan, mediante ese derecho de todos a la educación mostrando la oportunidad de ser educados.

Por consiguiente, debemos subrayar una serie de declaraciones que indican que el niño como todo ser humano tiene ya una serie de derechos, como la Declaración de Ginebra del 24 de septiembre de 1924, adoptada por la Asamblea de la Sociedad de Naciones Unidas destacando aspectos tan importantes, como son:

- Principio V: El niño física o mentalmente impedido o con algún tipo de impedimento social debe recibir el tratamiento, educación y cuidados especiales que requiere su caso en particular.

- Principio VII: EI niño tiene derecho a recibir educación, que será gratuita y obligatoria por lo menos en las etapas elementales. Se dará una educación que favorezca su cultura general y le permita, en condiciones de igualdad de oportunidades, desarrollar sus aptitudes y su juicio individual, su sentido de responsabilidad moral y social, así como llegar a ser un miembro útil de la sociedad. 
Posteriormente, en 1959 queda proclamada la Declaración de los Derechos del Niño. En ella se promulgan una serie de principios relacionados con la Pedagogía Hospitalaria, como son:

- Principio 4: El niño debe gozar de los beneficios de la seguridad social. Tendrá derecho a crecer y desarrollarse en buena salud, con este fin deberán proporcionarse, tanto a él como a su madre cuidados especiales, incluso atención prenatal y postnatal. El niño tendrá derecho a disfrutar de alimentación, vivienda, recreo y servicios médicos adecuados.

- Principio 5: El niño física o mentalmente impedido o que sufra algún impedimento social debe recibir el tratamiento, la educación y el cuidado especial que requiere su caso particular.

- Principio 7: El niño tiene derecho a recibir educación que será gratuita y obligatoria por lo menos en las etapas elementales. Se le dará una educación que favorezca su cultura general y le permita en condiciones de igualdad de oportunidades, desarrollar aptitudes y su juicio individual, su sentido de responsabilidad moral y social, y llegar a ser un miembro útil de la sociedad. El interés superior del niño debe ser el principio rector de quienes tienen la responsabilidad de su educación y orientación; dicha responsabilidad incumbe, en primer término, a sus padres. El niño debe disfrutar plenamente de juegos y recreaciones, los cuales deben estar perseguidos por la educación; la sociedad y las autoridades públicas se esforzarán por promover el goce de este derecho.

- Principio 8: El niño debe, en todas las circunstancias, figurar entre los primeros que reciben protección y socorro.

Más adelante, con la Convención de Naciones Unidas sobre los Derechos del Niño de 20 de noviembre de 1989, y adoptada por la Asamblea General y ratificada por 191 de sus 193 estados miembros, subraya una serie de derechos los cuales reconocen a todo niño el acceso a una serie de servicios como los educativos, de salud y servicios educativos apropiados a su edad, momento evolutivo donde tendrán derecho a ser escuchados e informados. En alguno de sus artículos se establece:

Artículo 1: se entiende por niño todo ser humano menor de dieciocho años de edad, salvo que en virtud de la ley que le sea aplicable haya alcanzado antes la mayoría de edad.

Artículo 23: en atención a las necesidades especiales del niño impedido, la asistencia que se presta será gratuita siempre que sea posible, y estará destinada a asegurar que el niño impedido tenga un acceso efectivo a la educación, la capacitación, los servicios sanitarios, los servicios de rehabilitación, la preparación para el empleo y las oportunidades de esparcimiento, y reciba tales servicios con el objeto de que el niño logre la integración y el desarrollo individual, incluido su desarrollo cultural y espiritual, en la máxima medida posible.

Artículo 28: Los Estados partes reconocen el derecho del niño a la educación a fin de que se pueda ejercer progresivamente y en condiciones de igualdad de oportunidades ese derecho, por ello se deberá:

- Implantar la Enseñanza Primaria obligatoria y gratuita para todos.

- Fomentar el desarrollo de la Enseñanza Secundaria, incluida la enseñanza general y profesional, hacer que todos los niños dispongan de ella y tengan acceso a ella, y adoptar medidas apropiadas tales como la implantación de la enseñanza gratuita y la concesión de asistencia financiera en caso de necesidad.

- Hacer la Enseñanza Superior accesible a todos.

- Hacer que todos los niños dispongan de información y orientación en cuestiones educacionales y profesionales y tengan acceso a ellas.

- Adoptar medidas para fomentar la asistencia regular a las escuelas y reducir las tasas de deserción escolar.

Tras haber mostrado estos Tratados, que muestran derechos y principios relacionados directa o indirectamente con nuestro ámbito de estudio vamos a destacar otro tipo de documentos muy 
significativos en nuestro sector, como son: La Carta Europea de los Derechos de Los Niños Hospitalizados aprobada el 13 de mayo de 1986, por Resolución del Parlamento (Diario Oficial de las Comunidades Europeas $n^{\circ}$ C.148/37), abogando el punto 4 donde se proclamen 23 derechos de los cuales hemos destacado los más prominentes:

- Derecho del niño a no ser hospitalizado sino en el caso de no poder recibir los cuidados necesarios, y en las mismas condiciones, en su casa o en su consultorio.

- Derecho a estar acompañado de sus padres, o de la persona que los sustituya, el máximo tiempo posible durante su permanencia en el hospital, no como espectadores pasivos sino como elementos activos de la vida hospitalaria.

- Derecho de sus padres, o de la persona que los sustituya, a recibir todas las informaciones relativas a la enfermedad y al bienestar del niño, siempre y cuando el derecho fundamental de éste al respeto de su intimidad no se vea afectado por ello.

- Derecho a disponer de locales amueblados y equipados, de modo que respondan a sus necesidades en materia de cuidados, de educación, juegos, así como las normas oficiales de seguridad.

- Derecho a proseguir con su formación escolar durante el ingreso y a beneficiarse de las enseñanzas de los profesionales de la educación y del material didáctico que las autoridades escolares pongan a su disposición.

En este sentido, Lizasoáin (2000) señaló ya en su momento que estos derechos hacen referencia a una serie de orientaciones, ya que no se trata de derechos en el sentido jurídico, sino que esta Carta fija unos objetivos fundamentados en una serie de principios, como pueden ser:

- Cubrir las necesidades de los niños hospitalizados, las de sus padres y todo lo que envuelve al niño enfermo.

- Lograr la adecuación de los servicios de salud.

- Preparar a los padres sobre el papel que ellos van a desempeñar en el Hospital.

- Preparar a los niños para su hospitalización.

Por otra parte, y remitiendo a la Carta de Derechos del Niño Hospitalizado de 1988 de la European Association for Children in Hospital (EACH) en Leiden (Holanda) se recogen diez derechos del niño hospitalizado, entre los que destacamos los más significativos:

- Los niños serán hospitalizados sólo en el caso de no poder recibir los cuidados que precisan en su casa o en un consultorio.

- Tendrán el derecho a estar acompañados durante todo el tiempo por sus padres o la persona que los sustituya.

- Los niños y sus padres tendrán el derecho a ser informados de manera apropiada a su edad y grado de comprensión. Se ofertarán las medidas necesarias para reducir su estrés físico y psicológico.

- Los niños tendrán plenas oportunidades para el juego, las actividades recreativas y educativas que sean adecuadas a su edad y entorno diseñado, amueblado, atendido y equipado en función de sus necesidades.

- Los niños serán atendidos por personal cualificado, con una formación y unas aptitudes que les permitan dar respuesta a las necesidades físicas, emocionales y de desarrollo, tanto de los niños como de sus familiares.

Finalmente, el 20 de mayo del 2000 la Hospital Organisation of Pedagogues in Europe (HOPE) presenta la Carta Europea sobre el Derecho a la Atención Educativa de los Niños y Adolescentes Enfermos aprobada por la Asamblea General de la Asociación HOPE, en el IV Congrés Europeu de Mestres i Pedagogs a l'Hospital en Barcelona en el año 2000 señalando derechos como: 
— Todo niño y adolescente enfermo tiene derecho a recibir atención educativa, tanto en el hospital como en su domicilio.

- El objetivo de esta educación es asegurar la continuación de la enseñanza escolar de los niños y adolescentes enfermos con el fin de mantener su rol de alumnos.

- La enseñanza escolar estructura la creación de un grupo de niños y de adolescentes, y normaliza la vida diaria en el hospital. La atención educativa se organizará de manera grupal o individual tanto en el aula como en la habitación del niño.

- La atención educativa en el hospital y en el domicilio deberá adaptarse a las necesidades y capacidades de cada niño o adolescente, manteniendo la conexión con su colegio de referencia para preparar su regreso.

- El aula hospitalaria, el entorno y los materiales de aprendizaje estarán adaptados a las necesidades de los niños y adolescentes enfermos. Las nuevas tecnologías de la comunicación servirán también para evitar el aislamiento.

- La enseñanza sobrepasará el contenido específico del currículo ordinario, incluyendo temas relacionados con las necesidades específicas derivadas de la enfermedad y de la hospitalización. Deberán utilizarse gran variedad de metodologías y recursos de aprendizaje.

- La atención educativa en el hospital y a domicilio correrá a cargo de personal cualificado que recibirá cursos de formación continua.

- El personal encargado de la actividad educativa formará parte del equipo multidisciplinar que atiende al niño o adolescente enfermo, actuando como vínculo de unión entre éste y su colegio de referencia.

- Los padres serán informados sobre el derecho de sus hijos enfermos a recibir atención educativa y acerca del programa educativo seguido. Serán considerados como parte activa y responsable en el mismo.

— La integridad del alumno será respetada, incluyendo el secreto profesional y el respeto a sus convicciones.

Con este aspecto compartimos que, el poder disfrutar de un derecho supone exigirle al Estado que lo cumpla, ello supone tener la obligación de respetar, así como proteger y llevar a cabo los Derechos Humanos que figuran en Pactos y Tratados Internacionales.

Por consiguiente y, para dar el paso al siguiente apartado, volvemos a destacar que el sector de la Pedagogía encargado de llevar a cabo este derecho en personas enfermas y hospitalizadas es, la Pedagogía Hospitalaria (Lizasoáin, 2000).

\section{DISCUSIÓN Y CONCLUSIONES}

Cuando hablamos de los Derechos del Niño hacemos referencia a esos logros y esfuerzos que se han alcanzado en los países desarrollados y se están consiguiendo en los países en desarrollo.

Gracias a la presencia de estas acciones educativas desarrolladas por la Pedagogía Hospitalaria se pueden reflejar los logros conseguidos. Por otra parte, queda reflejado que los sectores educativo y sanitario pueden trabajar juntos desarrollando equipos multidisciplinares que son los que consiguen dichos esfuerzos.

Por tanto, podemos afirmar que llevamos trabajando décadas en el proceso de enseñanza-aprendizaje del alumnado hospitalizado o convaleciente. Así pues, gracias a la Pedagogía Hospitalaria y a sus aulas se ha recorrido un largo trayecto, pero todavía queda mucho por recorrer hasta conseguir todos los objetivos planteados.

Todavía hay mucho por hacer en los países en desarrollo como: implantar tecnologías en las unidades escolares de apoyo; la Atención Educativa Domiciliaria ha de ser más rápida ante la demanda y, los derechos reflejados en Declaraciones Internacionales y en documentos oficiales puedan llegar a todo escolar que se encuentre ingresado o convaleciente. 
Todavía hay que progresar mucho en este sector fomentando la interacción de los campos multidisciplinares en los que se mueve: el educativo y el sanitario. Por ello, finalmente y confirmado está que, los países latinoamericanos están avanzando a pasos agigantados dentro del ámbito educativo hospitalario. Por tanto, recorrer un buen camino sobre las aulas hospitalarias y sus derechos supone trabajar juntos dentro de un equipo multidisciplinar.

\section{REFERENCIAS BIBLIOGRÁFICAS}

Calvo, I. (2017). La pedagogía hospitalaria:clave en la atención al niño enfermo y hospitalizado y su derecho a la educación. Aula 23, Ediciones Universidad Salamanca, pp.33-47.

Comunidad Europea (1986). Carta Europea de los Niños Hospitalizados. Diario Oficial de las Comunidades Europeas, No.148,148, 37-38. Resolución de 13 de mayo (doc. A2-25/86).

European Association for Children in Hospital-EACH (1988). Carta de Derechos del Niño Hospitalizado. Leiden, Holanda.

Lizasoáin, O. (2000). Educando al niño enfermo. Perspectivas de la Pedagogía Hospitalaria. Pamplona: Ediciones Eunate.

Molina, M.C. (2017). La Pedagogía Hospitalaria contribuye a la humanización de la atención sanitaria. Revista de Educación. Recuperado de http://www.revistadeeducacion.cl/felipe-ramirez-director-y-docente-de-la-escuela-hospitalaria-de-puerto-montt-este-es-un-trabajo-desde-el-corazon el 21 de diciembre de 2017.

Naciones Unidas (1959). Declaración de los Derechos del Niño. Recuperada de http://www.juridicas.unam.mx/publica/librev/ rev/derhum/cont/4/pr/pr20.pdf.

Naciones Unidas (1989). Convención sobre los Derechos del Niño. Recuperada de http://www2ohchr.org/spanish/law/index.htm el 28 de marzo de 2014.

Hospital Organisation for Pedagogues in Europe-H.O.P.E. (2000). Carta Europea sobre el Derecho a la Atención Educativa de los Niños y Adolescentes Enfermos. Asamblea General de la Asociación HOPE. Barcelona.

Torralba, F. (2002). Educar desde la vulnerabilidad. Actitudes éticas y excelencia profesional. IV Congrés Europeu de Mestres i Pedagogs a I'Hospital. ACPEAH. El futur de la pedagogía hospitalaria, p.13 Barcelona: Pau Education. ISBN 84-8294-593-9.

Sociedad de Naciones (SDN). Declaración de Ginebra sobre los Derechos del Niño, 26 de septiembre de 1924. Recuperada de http://www.humanium.org/es/ginebra-1924 el 17 de marzo de 2014. 